\title{
Embedded Web Server for Wireless Sensor Networks
}

\author{
Dejan Raskovic, Venkatramana Revuri, David Giessel \\ University of Alaska Fairbanks \\ Electrical and Computer Engineering Department \\ Fairbanks, AK, USA \\ d.raskovic@uaf.edu
}

\author{
Aleksandar Milenkovic \\ University of Alabama in Huntsville \\ Department of Electrical and Computer Engineering \\ Huntsville, AL, USA
}

\begin{abstract}
This paper presents an implementation of a platformindependent embedded web server and its integration into a network of wireless sensor nodes. The embedded web server is designed and built as an expansion module for one of the nodes in the wireless sensor network (WSN). It allows authorized Internet users to establish two-way communication with the sensor network. The server uses limited available hardware resources to implement an interface to the WSN node and to serve dynamic HTML pages to the remote user. This allows the user to monitor the operation of the WSN remotely, to periodically download the sensed data, and to change the operation mode of the network. In addition to providing monitoring and data collection services, the embedded web server can generate email alerts about critical issues in the WSN, provide secure access to modules that change the operation of the WSN, shut down sensor nodes, and log data from the network into an on-board flash memory.
\end{abstract}

Keywords- wireless sensor networks; embedded web server; remote monitoring and control

\section{INTRODUCTION}

Conventional data collection and monitoring methods, which require physical presence at the research site, could in many cases be replaced by remote monitoring over the Internet. This requires the Internet infrastructure to be available at the site, and that some of the devices in the data monitoring and collection network are web-enabled. A web-enabled device has a web server either integrated during the design of the device or added later as an expansion board. To distinguish such a web server from its full-featured, personal computer based cousin, we call it an embedded web server (EWS). The name should also stress the fact that such a web server, in most cases, has limited computational, storage, and energy resources available. Despite its hardware shortcomings, the EWS is expected to implement many of the services available on regular web servers. It has to be able to receive requests and commands from a remote Hypertext Transfer Protocol (HTTP) web client. It also has to be able to send the control and management interface of the web-enabled device in the form of Hypertext

This material is based upon work supported by the National Consortium for MASINT Research (NCMR) under Defense Intelligence Agency (DIA) / NSF Grant No. IIS-0434156. Any opinions, findings, and conclusions or recommendations expressed in this material are those of the authors and do not necessarily reflect the views of the NSF or DIA.
Markup Language (HTML) pages, through which the user can monitor and control the device.

A web-enabled network device allows the user to access the device using user friendly, platform independent and universally available web browsers. This eliminates the need for developing client software and it does not require the user to install additional software on her or his computer. Unlike the standard web browser, an embedded web server must have a relatively small footprint, both in terms of physical size and software size, to allow its easy integration into many devices. The user management interface should consist of standard HTML constructs, objects and images, and allow the user to interact with the device over the Internet. The device home page should provide the central navigation point to the device controls and data. The device should respond to user actions involving web page buttons, hot links, and other familiar browser controls.

This paper describes an EWS built primarily to serve as a remote access and control interface to wireless sensor networks (WSN). The constraints in terms of available energy, storage and computational resources can even be more stringent in this case. Fortunately, some of the features that are almost invariably required of regular web servers (such as the ability to serve multimedia content) are not considered to be crucial in the case of embedded servers for WSN. However, such a server will still have to be able to serve dynamic HTML pages, provide two-way communication, and to deal with large amounts of data. In addition, it will be required to implement two different communication protocols - one to establish the connection with the Internet and the other one to communicate with the WSN nodes.

\section{A. Related Research}

There is a number of interesting research efforts in the area of embedded web servers, and only a few can be mentioned here. The POStech - Embedded Web Server (POS-EWS) is an HTTP/1.1 compliant embedded web server implemented on the Xinu OS using the MPC 860 processor. The Hewlett Packard Laboratory - Cooltown project is exploring the convergence of web technology, wireless networks, and portable client devices through an infrastructure to support "web presence" for people, places, and things. The WebChip is a TCP/IPv6 and HTTP/1.1 
compatible, functionally minimized embedded web server for appliances. TargetWeb is an embedded web server for control and monitoring of embedded applications, designed to run on virtually any 32-bit CPU or DSP architecture. Tmote ${ }^{\mathrm{TM}}$ Connect is a wireless gateway application used to access Tmote wireless sensor modules through Ethernet. An Internetbased remotely accessible Hardware-In-the-Loop Simulator (HILS) for robotics and mechatronics applications has been developed at the University of Alaska Fairbanks .

\section{Motivation}

Fig. 1 shows the general overview of a typical WSN developed in the SensE Laboratory at the University of Alaska Fairbanks. Our group is developing mostly cluster-based energy efficient WSNs for monitoring, data collection, and surveillance. The majority of our applications require the network to be completely autonomous and intelligent enough to make decisions concerning mode of operation changes on its own. However, we occasionally need to monitor the network operation and collect the data for relatively short periods of time for offline processing and network analysis. The motivation for the development of the EWS was the need to allow on-demand, Internet-based insight into the operation of the sensor network and the data that has been collected, as well as to allow authorized users to change the operation mode of the sensor network.

Because we are working on several different wireless sensor platforms we needed the EWS to be platform independent. Therefore, we prefer the server to be a separate device that can be used as an expansion board for the existing sensor nodes. One of the sensor nodes of a currently monitored network is attached (using a standard serial connection) to the web server. In our terminology this node is called a EWS node. The EWS node is running the same software and implementing the same communication protocol as the rest of the sensor network nodes. The only difference is that, in addition to its normal functions, it can also forward messages from the EWS to the central node of the network and vice versa. Various infrastructure scenarios called for the web server capable of working with both wired (Ethernet) and wireless (802.11b) interfaces. Also, we needed flexibility in obtaining the IP address for the EWS. To allow access from off-campus locations we needed the server to be able to use a fixed, pre-

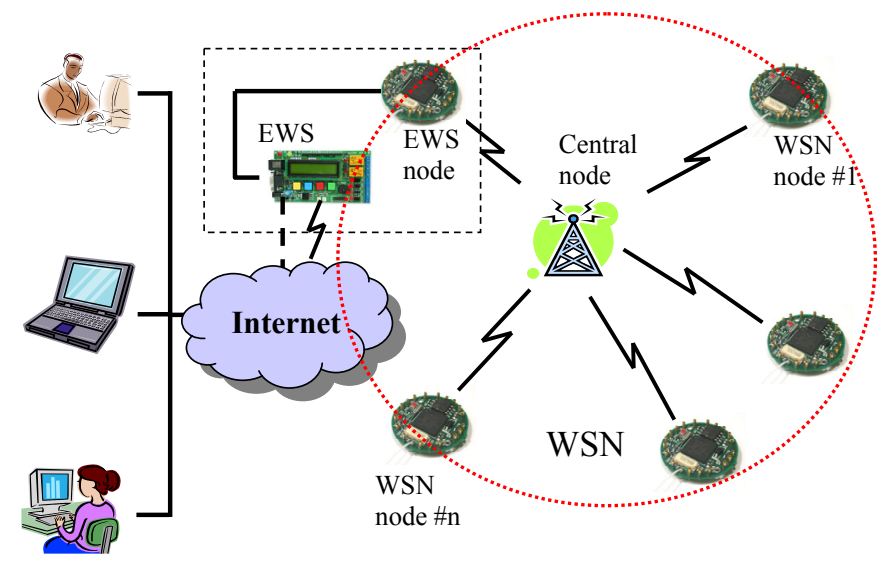

Figure 1. The general block diagram of a web enabled WSN

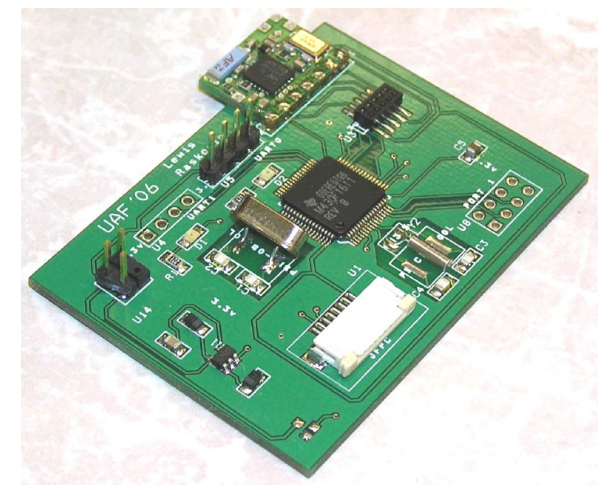

Figure 2. A prototype of WSN node

assigned IP address that is visible from the other side of the University firewall. For on-campus wired or wireless applications we needed the web server to be able to obtain an IP address from the DHCP server. Finally, for periodic visits to the WSNs employed in environments without Internet infrastructure, we needed the server to be able to operate in $\mathrm{Ad}$ hoc mode by establishing point to point connection with a WiFi enabled Personal Digital Assistant (PDA) or a notebook.

Our current wireless sensor node (Fig. 2) is based on a MSP430F1611 microcontroller from Texas Instruments (TI) and a $2.4 \mathrm{GHz}$ transceiver from Nordic Semiconductor with a maximum data rate of $1 \mathrm{Mbps}$. This prototype platform is used both as a sensor node (with the sensors attached to an expansion port) and as a central node (with additional flash memory and an optional serial link to a PC for debugging and data collection). The sensor nodes periodically wake up from the low power mode to sample the environment and to communicate with the central node using a custom developed energy efficient protocol. Each sensor node performs processing of data and sends the processed data to the central node in the assigned time slot. More details about the design and implementation of the sensor node, WSN organization, and the energy efficient protocol can be found in .

It is important to note that if the Internet-based data collection is expected to be the primary mode of data collection then the EWS should be interfaced directly with the central node. Such a solution would not be more difficult to implement, but it would be much more energy efficient because it would reduce the amount of data being transferred in the network. However, the main objective of our project was to design a portable and flexible solution that will allow periodic monitoring of the WSN operation, occasional changes of the operational modes, and intermittent data collection.

\section{EMBEDDED WEB SERVER REQUIREMENTS}

The main function of an EWS for WSN is to establish a connection with the sensor network in order to provide access to the data collected by the sensors and to allow the registered user to interact with the network. The user should be able to access the operational parameters of the network and to control the operation of the network by changing some of the parameters. The interface is implemented and the collected data is displayed through a series of HTML-based pages. Those pages reside in the flash memory of the EWS. The size of the 
available memory will determine the complexity of the user interface, the number of concurrent users that will be able to access the web server, etc. When a user enters the IP address of the EWS, the server typically needs to copy the appropriate HTML page into the random access memory (RAM), to embed the data in the form of dynamic content into that page, and to send the final page to the browser. Therefore, the amount of available RAM, which is one of the limitations of many microcontrollers, will play an important role.

The EWS also has to be able to store the collected data in the non-volatile memory (typically, flash memory) for network data analysis. The amount of available memory storage will directly determine how long the embedded server will be able to collect and store data and indirectly, the frequency and the resolution at which the data will be sampled by the sensor network.

Another issue of concern is the security in the device configuration and data monitoring. The EWS has to provide administrative privileges to permit access to certain network data and operational parameters only to authorized users.

\section{HARDWARE PlATFORMS AND INTERFACING}

\section{A. The Initial Solution}

All the sensor nodes in our WSN are custom built and based on microcontrollers from the TI MSP430 family of devices. Therefore, our first choice was to use a MSP430-based solution for a EWS. In that first phase of our project as a hardware platform we used a board developed by Olimex Limited, based on an MSP430F149 microcontroller and a CS8900A Ethernet chip from Crystal Semiconductor Corp. As a starting point for the firmware developed for this platform we used the basic TCP/IP implementation provided by TI and extended its capabilities to allow two-way communication. This solution is fully functional, but the TCP/IP stack and the web server itself have many limitations. The limitations, mostly caused by the limited hardware capabilities of the MSP430 microcontroller, include: only one open connection at a time, no virtual file system, no images, and very limited size of the web pages. However, this solution can still be very viable because of its very low energy consumption. All the details of this solution can be found in .

\section{B. An Improved Solution}

An improved solution is based on an RCM3750 RabbitCore $^{\mathrm{TM}}$ module developed by Rabbit semiconductor . This module features a Rabbit $3000 \AA$ microcontroller running at $22.1 \mathrm{MHz}$, with $512 \mathrm{~KB}$ of flash memory and $512 \mathrm{~KB}$ of SRAM memory. The module also includes a single-chip Ethernet controller from ASIX that implements 10/100Base-T connectivity and $1 \mathrm{MB}$ of serial flash memory. The physical connection between the Rabbit module and a WSN node is implemented using the standard UART interface. The communication protocol and its implementation will be briefly described in the following section. A more detailed description of the communication protocol can be found in $[8,9]$.

\section{SOFTWARE IMPLEMENTATION}

In addition to higher clock frequency and larger memory capacity, the RabbitCore offers some additional features that presented incentive for switching to this platform. The following table lists some of the advanced features and the benefits gained.

\begin{tabular}{|l|l|}
\hline FEATURE & ALLOWS... \\
\hline Increased security & Adding access control \\
\hline $\begin{array}{l}\text { SSI and CGI } \\
\text { support }\end{array}$ & $\begin{array}{l}\text { Inclusion of complex dynamic data into the } \\
\text { response from sensor nodes }\end{array}$ \\
\hline $\begin{array}{l}\text { Support for virtual } \\
\text { file system }\end{array}$ & $\begin{array}{l}\text { Inclusion of images and improvised } \\
\text { organization of file system and storage }\end{array}$ \\
\hline Dynamic C & $\begin{array}{l}\text { Easy application development using an } \\
\text { advanced TCP/IP stack library }\end{array}$ \\
\hline $\begin{array}{l}\text { Support for web } \\
\text { compiler }\end{array}$ & $\begin{array}{l}\text { Simplifies the process of presenting the web } \\
\text { variables to the server }\end{array}$ \\
\hline
\end{tabular}

Exploiting the above advantages over the MSP430, we developed an application programming interface to implement a secure and reliable HTTP server. A set of firmware programs was developed to monitor and control the WSN, and to collect the data sensed by the nodes of the sensor network. The firmware includes the protocol developed to implement the communication between the web server and the network. The data collection and retrieving techniques are developed; further, an extensive web-based user management interface is designed and all the web server controls are connected to this interface. The user interface features various forms which allow the user to fill in the information that comprises a request and to submit it to the server. The server application performs the requested operation and sends the results to the client with the necessary dynamic content embedded into the initial web page.

The level of complexity of programming increases greatly with the increase in demand for the dynamic nature of the user management interface. Several other features which should be considered in developing a reliable and robust web server include: user authentication and access control, amount of configurable data uploaded to the server, number of web pages, number of controls on the web page, etc. Each feature needs an application program to handle the requirement.

\section{A. The Communication Protocol}

Communication between the remote client and the WSN is handled jointly by the EWS and the EWS node, through the HTTP server. The communication between the EWS node and the hub is outside of the scope of this paper. It is implemented as an extension of the regular communication protocol used by the specific WSN. The implementation details can be found in [9].

Fig. 3 shows the data format of a typical message exchanged in the network. In addition to several message types

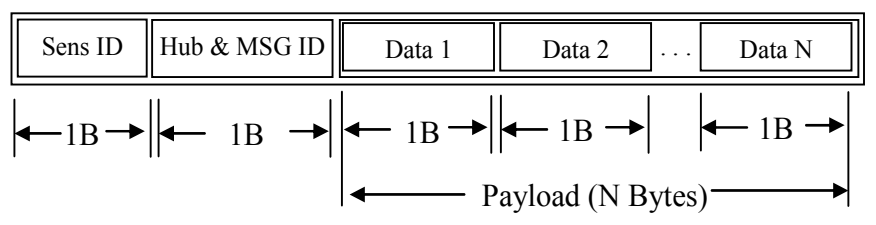

Figure 3. Message format 
used to collect the actual sensor data, the other types of messages are implemented to: obtain the list of currently active nodes; to change the node's radio bit rate, frequency channel, and the output power; to shut down a specific sensor node, etc. The exact format of the header and the number of bytes in the payload is variable and depends on the sensor network for which the EWS is used.

\section{B. Web Server Operating Modes}

The web server can operate in one of the following three modes: snapshot mode, monitoring mode, and log mode.

In the Snapshot mode the server passively listens to the requests for network parameters (data) from the clients. When the request is received, the server initiates communication with the EWS node. The embedded web server node forwards the request to the central node. The central node retrieves the most recently collected network data and responds to the EWS node which in turn forwards it to the server. The server processes the received data and presents the graphical representation of the parameter value to the client. Snapshot mode is used to display the current network data in near-real-time.

In the Monitoring mode the server continuously monitors the network and actively collects the data from the network, as configured. When the server is operating in monitoring mode, the result shows a continuous live parameter values using a graphical representation of the data. The time between the requests depends on the frame period of the WSN.

In the Log mode the server collects the request subset of network data for a given period of time. The data is stored in the EWS' serial flash. The data can be remotely read from the flash and decoded and displayed using an application developed in MATLAB ${ }^{\circledR}$. Depending on availability, the flash contents can be read either through the web browser or directly from the serial port of the web server. The RCM3750 has an Atmel Flash AT45DB081B, a serial interface $1 \mathrm{MB}$ flash memory designed specifically for code and data storage applications. In log mode, the server collects data from the network and stores it on the serial flash.

\section{EWS and WSN Controls}

The web server supports three groups of users unregistered users can access the server and see the data if the server is already in the monitoring mode; administrators have unrestricted access to all server and sensor network controls; registered users can change the operation mode of the server, request previously logged data, and have limited access to some of the embedded server controls.

A member of the administrator user group is allowed to access the WSN Controls. These pages allow various parameters of the radio (the number of address bytes, output power, communication channel, and the data bit rate) to be configured. Also, the administrator can select the types of data to be collected and the way the data are presented. The same pages allow a specific sensor node to be shut down.

The Web Server Controls allow an authorized user to change the operating mode of the server, erase the sessions with logged data from the flash memory, configure the email alerts, and set the time and date on the server.
An example of one of the configuration pages presented to a user from the administrator group is given in Fig. 4. The user has an option to set the mode of operation (snapshot, log mode, or monitoring mode), change the types of data to be monitored or logged, change the data capture duration, or request the previously logged data.

The EWS is capable of sending an email to an administrator or an authorized email user when certain conditions are met. The alert configuration page allows the authorized user to set those conditions. Typical configurations include sending an email: if the server does not respond to configured number of requests from the web server, if the server's serial flash is full, if the sensor parameter values reach the set threshold. The server generates the appropriate subject and body of the email describing the reason for the email alert. It also includes a dynamically generated email body identifying the sensor node(s) which reached the set threshold. This feature keeps the administrator informed about the network status and the data collected by the network.

\section{Client- and Server-Side Scripting}

We used client-side and server-side scripting to add dynamic behavior, visual effects and form validation to HTML pages served by the EWS. The scripts are typically mixed with the HTML code of the web document. HTML provides the user-interface and the scripts provide the logic to govern the dynamic behavior of the document. The JavaScript is used to create a dynamic, interactive web-based application that runs entirely within a browser. Using JavaScript to add dynamic features to the web pages, such as reacting to user events, comes with some disadvantages. A browser with disabled or not implemented JavaScript processing will not be able to display the entire page correctly. Subtle differences in browser implementations can cause slight errors, possibly affecting the display or even the functionality of the user interface. However, this disadvantage is restricted to that particular client machine and does not affect the web server operations or other client machine's web page display. EWS implementation also uses a large number of Server Side Include (SSI) and Common

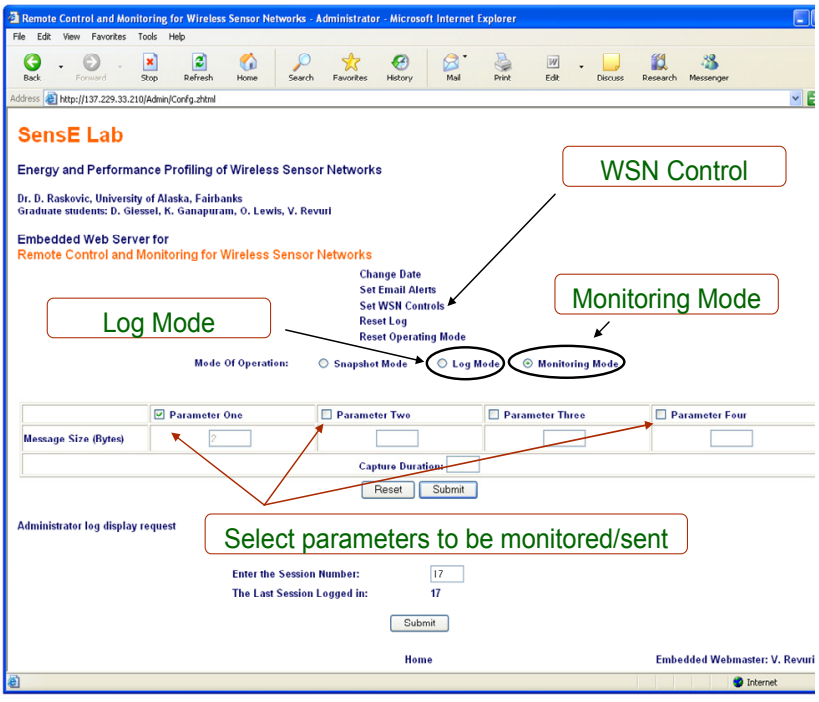

Figure 4. One of the configuration pages in the Administrator domain 


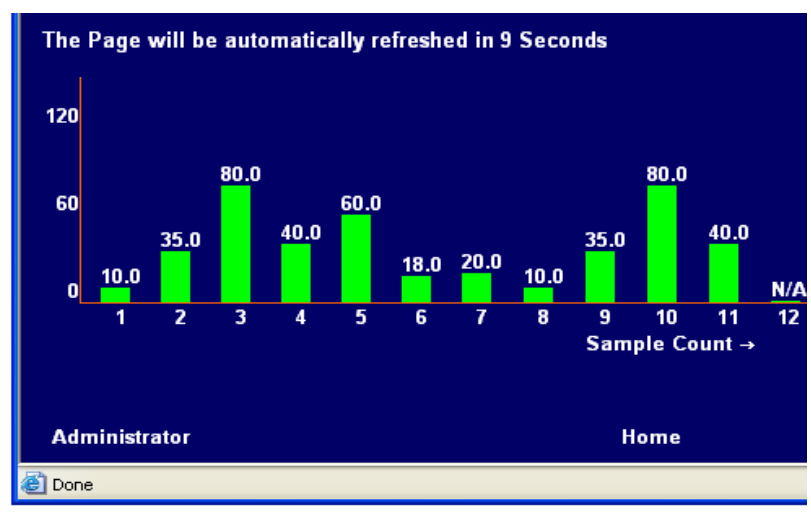

Figure 5 . The page served by the EWS working in the monitoring mode. The page is automatically refreshed to display new data

Gateway Interface (CGI) functions. In addition, the RabbitWeb, an add-on module for Dynamic C, uses a SSI-like scripting language. Fig. 5 shows a portion of the page generated when a user sends a request to monitor one of the parameters sent by one of the sensors over a given time interval. The data is displayed in a form of a sliding bar graph.

\section{E. Email Alerts}

The EWS is capable of sending an email to an authorized user from the web server in the case of the following events: the server stops responding, the server's serial flash is full, or if the monitored data reaches the preset $\min / \max$ thresholds.

\section{F. Wireless Internet Connectivity}

An optional kit adds wireless Internet connectivity to the RCM3750 RabbitCore web server. This kit enables the EWS to operate in one of the two modes: an infrastructure mode or an ad-hoc mode.

The EWS operates in an infrastructure mode when the infrastructure is available in the EWS vicinity, allowing the client to access the server from any place where the Internet connectivity is available. In the ad-hoc mode the EWS establishes a peer to peer connection with any $802.11 \mathrm{~b}$ enabled device that has a browser installed. This mode of operation is suitable for periodic control or data collection visits to remote WSN sites where Internet infrastructure does not exist. Fig. 6 shows a EWS with a wireless module working in the ad-hoc mode and serving pages to a Hewlett Packard iPAQ hx4700 PDA. When operating in infrastructure mode the Rabbit web server either issues a request to the DHCP server for an IP address or uses a previously assigned static one. If the DHCP server responds and assigns a valid IP address to the EWS, the server sends an email with this address to the administrator.

\section{CONCLUSION AND FUTURE WORK}

We developed a flexible, embedded web server for monitoring, data collection, and control of WSNs. The server uses one of the sensor nodes to join the network and provide an authorized user access to a remote network of sensor nodes. The flexibility and portability of the system allows us to use it with sensor networks implemented using different hardware

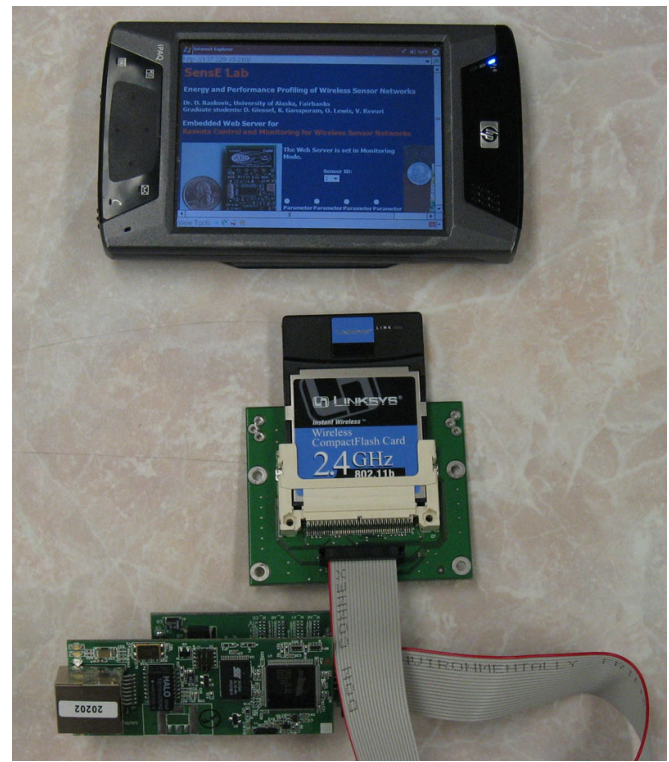

Figure 6. The EWS working in an Ad-Hoc wireless mode

platforms. It can also be easily changed to accommodate applications that do not even require the use of WSN. For example, we are currently testing a configuration that uses the EWS to provide the remote access to an array of temperature and humidity sensors to be used for the research of bugs capable of surviving extreme Alaskan winters.

\section{REFERENCES}

[1] J. Hong-Taek, C. Mi-Joung, and J. W. Hong. (2000). "An Efficient and Lightweight Embedded Web Server for Web-based Network Element Management," International Journal of Network Management. 10, pp. 261-275.

[2] K. Tim et. all., "People, Places, Things: Web Presence for the Real World," ACM MONET (Mobile Networks \& Applications Journal). 7(5), pp. 365-376.

[3] R. Janne, M. Peri, M. J. Saaranen, R. Jussi and S. Juha-Pekka. (2001, October). "Providing Network Connectivity for Small Appliances: A Functionally Minimized Embedded Web Server," IEEE Communication Magazine.

[4] Blunk Microsystems - TargetWeb, http://www.blunkmicro.com/web.htm, September, 2006.

[5] Moteiv - Tmote Connect, "Tmote Connect - Data Sheet," http://www.moteiv.com/products/docs/tmote-connect-datasheet.pdf, February 2006.

[6] A. Turan, "Remotely Accessible Hardware-in-the-Loop Robot Simulator" MS Thesis, University of Alaska Fairbanks, Electrical and Computer Engineering, 2006.

[7] SensE Laboratory, ECE Department, University of Alaska Fairbanks. http://www.faculty.uaf.edu/ffdr/research.htm

[8] O. Lewis, "Time Synchronization and System Support for Energy Efficient WSNs," MS Thesis, University of Alaska Fairbanks, Electrical and Computer Engineering, 2007.

[9] V. Revuri, "Internet Based Data Collection and Monitoring for WSNs," MS Thesis, University of Alaska Fairbanks, Electrical and Computer Engineering, 2007.

[10] Rabbit Semiconductor, "RCM3750 RabbitCore Data Sheet," http://www.rabbitsemiconductor.com/products/rcm3750/rcm3750.pdf, August 2005. 